\title{
Validation of the State Version Questionnaire on Autonomic Regulation (State-AR) For Cancer Patients
}

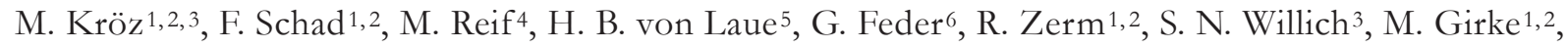 \\ B. Brinkhaus ${ }^{3}$ \\ ${ }^{1}$ Department of Internal Medicine and ${ }^{2}$ Research Institute Havelhöhe (FIH) at the Community Hospital Havelhöhe, Berlin; Germany, \\ ${ }^{3}$ Institute for Social Medicine, Epidemiology, and Health Economics Charité University Medical Center, Berlin, Germany, \\ ${ }^{4}$ Institute for Clinical Research IKF, Berlin, Germany, ${ }^{5}$ Humanus Institute, Berlin, Germany, \\ ${ }^{6}$ Academic Unit of Primary Care, University of Bristol, UK,
}

\begin{abstract}
Objectives: Current quality of life inventories used in oncology mainly measure the effects of chemo- or radiotherapy alongside functional and role scales. A new approach is to measure the autonomic state of regulation with the trait-inventory of autonomic regulation (Trait-aR). Loss of Trait-aR has been shown in different medical conditions such as breast cancer (BC) but not in colorectal cancer patients (CRC). In this paper we report the validation of a new state autonomic regulation scale (State-aR) of the last week.
\end{abstract}

Methods: Study 1 included 114 participants: (41 women/16 men with cancer and 57 age- and gendermatched healthy people) to conduct a reliability-, factor- and validity-analysis. Concurrent and convergent validity was evaluated with Trait-aR, Fatigue-Numerical-Scale, Hospital Anxiety and Depression Scale (HADS-D) and the self-regulation scale, 65 participants were retested. Study 2 completed 42 participants: 17 with $\mathrm{BC}$ and 25 with $\mathrm{CRC}$ receiving chemotherapy. The State-aR was administered prior, during and after chemotherapy for measuring responsiveness.

Results: The factor analysis loaded to four subscales of State-aR (rest-activity, orthostatic-circulatory, thermosweating and digestive regulation) with a: Cronbach- $\alpha$ $\mathrm{r}_{\alpha}=0.77-0.83$ and $\mathrm{a}$ test-retest-reliability $\mathrm{r}_{\mathrm{rt}}=$ $0.60-0.80$. The sum- and subscales correlated with their concurrent subscales in the Trait-aR $(0.48-0.74)$ and with the sum-scale moderately with all convergent criteria $(r=0.41--0.44 ; \mathrm{p}<0.001)$. During chemotherapy the State-aR-sum and rest-activity-scale decreased significantly compared to the change in the Trait-aR ( $p$ $<0.05)$.

Conclusions: These findings support that the state autonomic regulation scale has satisfactory to good reliability, good validity and acceptable responsiveness in the context of chemotherapy treatment.

Key words: Cancer, Cancer-related-fatigue, (Trait and State) autonomic regulation (aR), health related quality of life, validation study

List of Abbreviations: $\mathrm{BC}=$ breast cancer, $\mathrm{CMF}=$
Cyclophosphamide, metotrexate, 5-Fluor-uracile, CRC $=$ colorectal cancer patients, FEC $=5$-Fluor-uracile, epirubicine, cyclophospamide, FOLFOX $=5$-Fluoruracile, oxaliplatine, HADS-D = Hospital Anxiety and Depression Scale (German Version), OXALI = oxaliplatine mono, $\mathrm{RAD}=$ radiotherapy, State $\mathrm{aR}=$ state autonomic regulation, Tac $=$ Taxole, Trait $a \mathrm{R}=$ trait inventory of autonomic regulation

\section{INTRODUCTION}

Generic and condition specific health related quality of life (HRQL) questionnaires, such as the SF-36 and EORTC QLQ-C30 or FACT respectively, that assess physical and psychosocial functioning are commonly used in oncological practice and research $[1,2,3]$. The cancer specific scales have additional measures to detect the adverse effects of chemo- or radiotherapy [2, 3].

Interviews with cancer patients have found a broader range of factors influencing quality of life than is captured in current HRQL measures [4, 5, 6]. Cancer-related Fatigue Syndrome (CRF) is one of the most significant factors determining HRQL in cancer patients. 58\% of all outpatients [7] cite it as one of the most burdensome symptoms and it affects up to $92 \%$ of all patients undergoing chemotherapy [8]. $34 \%$ of breast cancer patients report fatigue 5 to 10 years after diagnosis [9]. Research on correlates of CRF open up new therapeutic approaches and opportunities for intervention $[10,11]$. For example, there is increasing research interest in sleep disturbances. A cross-sectional study of 982 cancer patients found sleep disorders in $31 \%$ of patients [12] and in diseasefree breast cancer patients $46 \%$ reported these symptoms between one to three years after initial diagnosis [13]. During chemotherapy 59.5\% out of 991 patients reported sleep disturbances, of which $28.5 \%$ meet the diagnostic criteria for an insomnia syndrome (in breast cancer patients: $69.6 \%$ and $36 \%$ respectively) [14]. In the case of patients with stage IV colorectal carcinoma actigraphic measurements show a disturbed rest- and reduced daily activity rhythm coincides not only with reduced HRQL but also with reduced survival time [15]. 
In oncological research to date there has been little investigation of autonomic regulation and functional disorders which are associated with sleep problems and general feelings of ill health [16], although in rehabilitation medicine the new international classification of functioning (ICF) clearly articulates autonomic and physical functions as important outcomes [17, 18]. Our concept of autonomic regulation (aR) encompasses individual autonomic functions and the rest-activity rhythm. Rudolf Steiner, the founder of anthroposophic medicine, highlighted in the 1920s the potential role of autonomic regulation in sustaining health and the development of illness [19]. After initial studies which did not result in sufficient reliability [20] [21] we developed a trait-marker questionnaire on autonomic regulation (initially referred to as endogenous regulation): trait-aR. Autonomic regulation (aR) refers to the state of regulation of different autonomic functions in the rhythmic cycle of rest and activity over the course of the day. aR is effected by constitution, gender, age and illness. Under physiological conditions it is a stable trait, but in the context of illness or psychological disturbance a loss of regulation can occur which leads to a decreased aR [22]. We have validated a short version of Trait-aR with 12 items capturing the rest-activity- and orthostatic-circulatory regulation [23] and a longer version with 18 items which includes digestive regulation, too [22].

Loss of aR occurs in patients with a range of medical conditions, including some cancers [24, 25]. Cohen $\&$ Mount focus on the important issue of "good" days and "bad" days for patients with cancer and our relative ignorance about what drives this variation [26]. With regards to aR this means that the frequently changing condition of patients with metastatic cancer or of those receiving oncological treatment is possibly not being sufficiently measured with the Trait-aR scale, which captures autonomic constitution together with current regulation. The aim of the two linked studies we report in this paper was to develop and validate a questionnaire on autonomic regulation referring to the previous week (State-aR) which captures the current state of regulation more precisely and to distinguish between constitutional autonomic regulation and loss of regulation due to illness or side effects of treatment.

\section{Methods}

\section{SAmple Population}

In the first study we tested tool reliability, conducted a factor analysis and analysed concurrent validity. In the second study we measured responsiveness to change in the context of chemotherapy treatment.

\section{Study 1: Item Detection, ReLiability AND VALIDITY}

An expert panel using an informal consensus method identified 23 items from the trait version questionnaire on autonomic regulation that were judged relevant to the current (past week) autonomic state (see assessment instruments).

We carried out the first study in the departments of General Internal Medicine and Gastroenterology at the Gemeinschaftskrankenhaus Havelhöhe (Berlin), as well as in the hospital's specialist oncology practice between January 2003 and February 2004.

Consecutively recruited patients with histologically confirmed malignant tumours were matched by age ( \pm 5 years) and gender with participants from a healthy control group, recruited opportunistically from hospital staff and their families, who had no known acute or chronic condition. Table 1 shows the demographic profile Table 2 the clinical and treatment characteristics of the participants and Table 3 the range of malignancies in participating patients. At the time of questionnaire administration, at least two weeks had elapsed since a participant's last operation, chemotherapy or radiotherapy session. Exclusion criteria included patients with a Karnofsky Index (KPI) $<50 \%$, patients with a manifest psychosis or badly controlled pain and participants aged $<18$ years or $>$ 85 years.

Table 1. Sociodemographic data of patients and healthy controls of study 1 and 2.

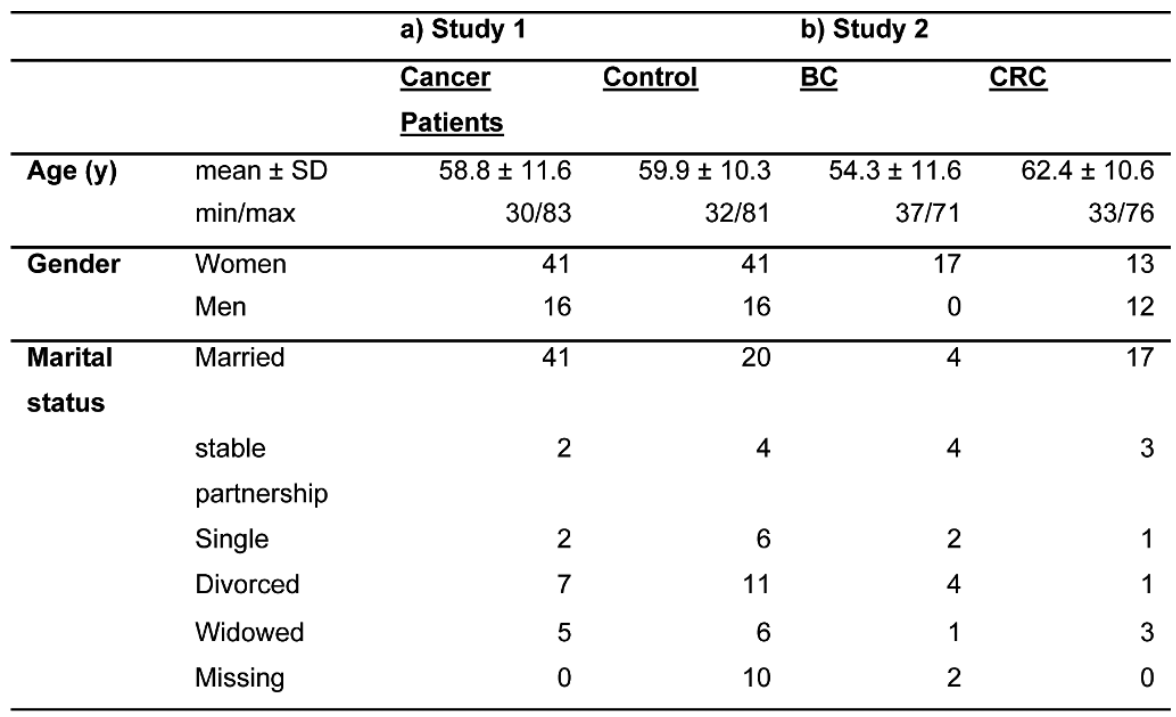


Table 2. Overview of tumour stage, diagnosis duration, Karnofsky performance index, haemoglobin and therapy.

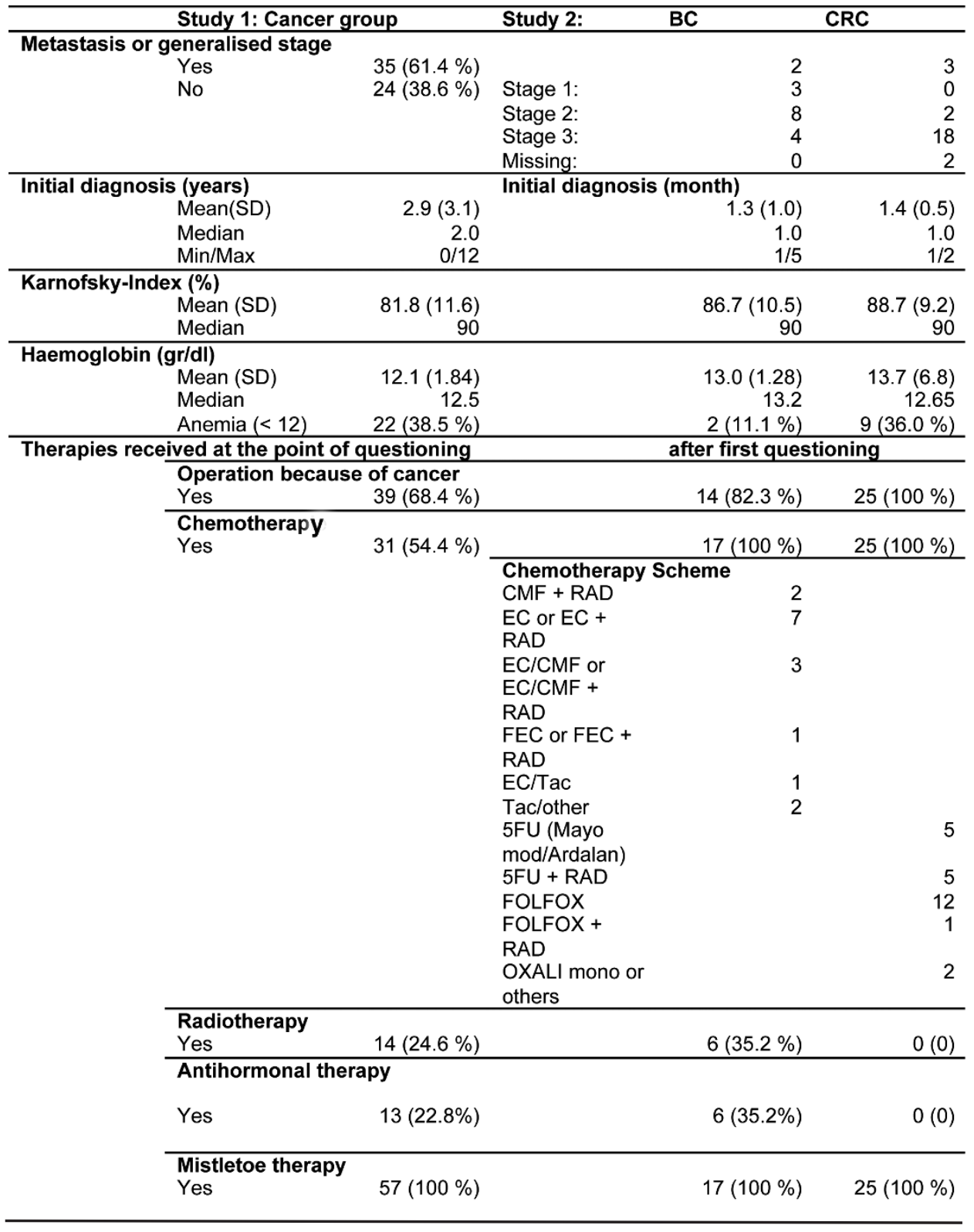

After the study was explained and consent was obtained, the State-aR questionnaire was administered. The target for the test-retest analysis was at least 50\% of all participants. The questionnaire was re-administered to participants 1) who were reviewed as in- or outpatients in our centres or 2) who consented to retest when they initially completed the questionnaire and were mailed the second questionnaire after a median of four weeks. All participants asked to complete the second questionnaire consented to do so.

\section{ASSESSMENT INSTRUMENTS}

A group of experts (oncologist, internist, gastroenterologist, sleep specialist, general practitioner and statistician) compiled 23 items largely derived from the Trait-aR scale with a clear focus on the clinically relevant last week, with topics of rest/activity, circadian well-being, motor skills, orthostatic and circulatory, di- gestive, thermo- and sweat regulation. The scale structure of the trait scale was retained as much as possible and items were re-worded in terms of the past week; 'travel sickness', 'dizziness from circular motions (e.g. when on a round-about)' could not be used in that context and were therefore replaced. The item 'cold and cold sweaty extremities' was split into 'cold extremities' and 'cold and sweaty extremities'. The following new items were included: 'fine motor skills', 'activity-dependent sweating', 'stool consistency' and 'vivid dreams' (Table 4). Each question has five possible answers which are rated with a five-point Likert scale from 1 to 5 ('not at all' to 'very'), or in the case of negative questioning, rated inversely (Table 4). The question relating to frequency of bowel movements offers different possible answers for the frequency of different stool consistencies.

Next to the 23 state-aR items (Table 4) the following questionnaires were included in the validation 
Table 3. Tumour diagnoses of patients with malignant tumours.

Included

Frequency Percents

B-chronic lymphatic leukaemia

(B-CLL)

B-cell Lymphoma (NHL)

Breast cancer

Bronchial carcinoma (NSCLC)

Carcinoma unclear primary

(CUP)

Cervix carcinoma

Colon carcinoma

Gallbladder carcinoma

Hypernephroma

Leiomyosarcoma

Melanoma

Ovarian carcinoma

Ovarian sarcoma

Pancreatic cancer

Pharynx cancer

Plasmocytoma

Pleuramesothelioma

Prostate cancer

Rectal carcinoma

Thymus gland carcinoma

Thyroid carcinoma

Tonsillar carcinoma

Urethral cancer

Total

study as congruence

$(2-6)$ :

(1) and convergent criteria

1) The Trait-aR questionnaire measuring the usual autonomic functioning of an individual with an 18item scale in three subscales (orthostatic-circulatory, rest/activity regulation and digestive). Trait-aR measures with a three-point Likert scale (18-54) with a satisfactory - good reliability and good validity. Higher scores indicate better aR. We used this scale to measure congruence and to validate the state/trait concept and to test a hypothesised difference in responsiveness [22].

2) A 16 item questionnaire measuring 'self-regulation' (SRS) developed by Grossarth-Maticek [27]. The questionnaire measures self-regulation and healthbuilding activity in two subscales with a six-point Likert scale. The 16 items are added and divided by 16 to obtain a total score $(1-6)$. Subscale 1 is termed "Ability to Change Behaviour in order to Reach Goals", and subscale 2 "Achieve Satisfaction and Well-Being", which therefore has a hedonistic connotation. Higher scores indicate better self-regulation. The validity and reliability of the sum- and subscales are good to very good. [27, 28]

3) The German version of the 'Hospital Anxiety and Depression Scale' (HADS-D) consisting of 14 items (7 for anxiety and 7 for depression) which people rate on a four-point Likert scale ( $0-21$ for both).
Higher scoring indicates more symptoms, $\geq 11$ points anxiety or depression is probable, $\geq 8-10$ possible cases, $<7$ no cases. The HADS is highly reliable and valid and is an extensively used scale in internal medicine research [29, 30].

4) The Fatigue-Numerical-Scale (FNS), an instrument measuring fatigue between 0 (no fatigue) and 10 (worst fatigue); $\geq 4$ stands for moderate, $\geq 7$ for severe fatigue $[31,32]$.

5) Single items regarding thermoregulation (ie. congestive perspiration or freezing) [23]

6) The Karnofsky Performance Index (KPI) which is a physician-assessed, general robust indicator of physical functioning in daily life [33].

Additionally for convergent validity, we documented the last haemoglobin level in the blood $(\mathrm{g} / \mathrm{dl})$ before inclusion of all patients with malignancies.

Results of the simultaneous validation of the German version of the Cancer Fatigue Scale (CFS-D) [34] and of the Internal Coherence Scale (ICS) [35] have been published elsewhere.

\section{StUdy 2: StATE-AR RESPONSIVENESS TO CHEMOTHERAPY TREATMENT}

From April 2003 to March 2007, the Centre for Tumour Therapy at the Gemeinschaftskrankenhaus Havelhöhe, the Oncological Practices in Öschelbronn and Havelhöhe carried out the second study to test responsiveness of the State-aR questionnaire in the context of chemotherapy treatment. After explanation of the procedure and obtaining informed consent, we recruited consecutive breast cancer (B) and colorectal cancer patients (C) with histologically proven tumours who received adjuvant or palliative chemotherapy and concomitant mistletoe therapy. The participants were between 18 and 85 years old with a KPI $>70 \%$ at baseline The oncologically scheduled chemotherapy, radiotherapy or mistletoe therapy was not impacted or changed by the study so that this second part of the validation study only measured the changing sensitivity (responsiveness) of the instruments. Therefore, study 2 is not a pharmacological study to test effectiveness of the treatment which was administered as a routine procedure in the study centres, but a validation and sensitivity testing of the instruments.

The State-aR responsiveness was compared to the Trait-aR version before, during and after adjuvant or palliative chemotherapy.

1) Breast cancer patients who received adjuvant complementary treatment with standardized whole plant extracts of Viscum album L. (mistletoe) as standard therapy from the first cycle of chemotherapy (B) or

2) Colorectal cancer (CRC) group (C) was also surveyed prior to the first cycle of chemotherapy with adjuvant mistletoe therapy.

For this purpose, the surveys were carried out 1-5 days prior to the start of chemotherapy (B, C), 1-5 days after the third cycle as well as $4-8$ weeks after the end of chemotherapy using the State-aR and TraitaR, and EORTC QLQ-C30 HRQL measure [2]. For KPI, stage of cancer, surgery and chemotherapy see Table 2. 
Table 4. The list of items of the State version on autonomic regulation (State aR) with the four subscales and the different answer possibilities and their scoring values. The items are arranged from top to bottom for State rest/activity, State orthostatic-circulatory, State digestive and State thermo-sweating regulation. List of the non-validated items with answers and scoring values.

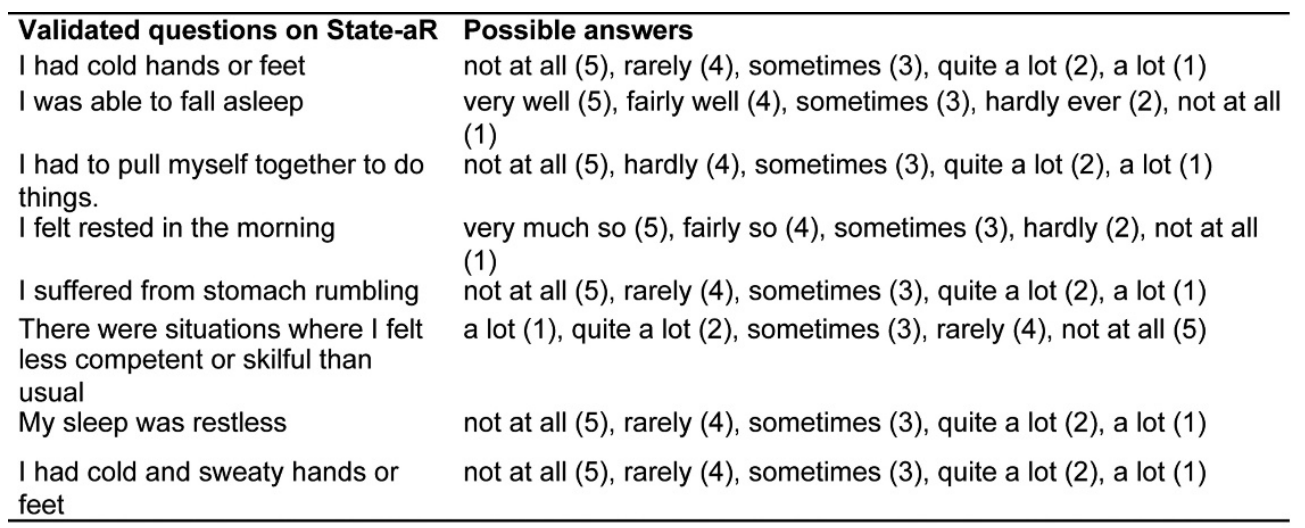

I felt dizzy when turning around I felt dizzy when I got up in the morning

I felt dizzy when turning around quickly

I felt dizzy when getting up or bending down

I suffered from night sweats I sweated

I sweated even during light physical activity

\section{I had bowel movements}

I had bowel movements - as always - at the same time(s) of the day

I suffered from constipation not at all (5), rarely (4), sometimes (3), quite a lot (2), a lot (1) not at all (5), rarely (4), sometimes (3), quite a lot (2), a lot (1)

not at all (5), rarely (4), not sure (3), quite a lot (2), a lot (1) not at all (5), rarely (4), sometimes (3), quite a lot (2), a lot (1) a lot (1), quite a lot (2), sometimes (3), rarely (4), not at all (5) a lot (1), quite a lot (2), sometimes (3), rarely (4), not at all (5) a lot (1), quite a lot (2), sometimes (3), rarely (4), not at all (5) 3 times/day (5), 2 times/day (4), 1 /day (3), 3-4 times/week (2), 1-2
times/week
not at all (1), to a small extend (2), don't know (3), to a large
extend (4), fully (5)
not at all (5), rarely (4), sometimes (3), quite a lot (2), a lot (1)

\section{I felt best}

I remembered dreaming My dreams were vivid

I was able to digest big meals

The stool consistency was: in the morning (5), best in the morning (4), at lunchtime (3), best in the evening (2), in the evening (1)

not at all (5), rarely (4), not sure (3), quite a lot (2), a lot (1)

very vivid (0), quite vivid (1), moderately so (2), hardly (3), not at all

(4), I cannot remember my dreams (5) very well (5), quite well (4), sometimes (3), not very well (2), not at all (1), I did not eat any big meals (0)

liquid (5), pasty (4), formed (3), very formed (2), hard (1)

\section{Statistical Analysis of Both Studies}

The primary outcome of the validation study was to develop a reliable, valid and sensitive questionnaire on autonomic regulation for cancer patients.

For this purpose we conducted a listwise first reliability analysis for all 23 items along the following criteria:

1) The Item-Total-Correlations being $\geq 0.10$ and $\leq 0.70$

2) The $\alpha$-if item deleted-analysis of every item should not achieve higher values than the Cronbach- $\alpha$ of every item.

3) The remaining items were examined as to sufficient item population.

4) Following that a reliability analysis (item-item-, item-total-, Cronbach- $\alpha$ - and test-retest-reliability) is carried out. Due to the concept-based heterogeneity of the items for different autonomic functions the following limits were determined for the sum-scale: Cronbach- $\alpha$ and test-retest-reliability: $\geq 0.70$.

5) The test-retest reliability was assessed with Spearman's rank correlation. The test-retest-reliability should be below that of the trait-scale.

6) On the basis of these pre-selected items for all participants a principal component analysis (rotation: Varimax with Kaiser normalization) was conducted with optimality assessment of the two-, three- fourand five-factor model respectively, at a minimum factor load of $\mathrm{r}=0.40$.

7) The Trait-aR questionnaire was used as a main convergence criterion. In addition, we performed a Spearman's rank correlation analysis for the whole sample, testing for associations between the State-aR sum- and subscales on the one hand and the assumed 
Table 5. Overview over the items of the State aR with their distribution over the different subscales: Arranged from top to bottom for State rest/activity, State orthostatic-circulatory, State digestive and State thermo-sweating regulation with details on mean (SD), minima/maxima and concurrent validity for item/total and item/subscale correlation with Trait aR sum scale. Furthermore, item-total-correlation, factor loading, $\alpha$-if item deleted for the subscales, Cronbach$\alpha$ and retest reliability are presented (significant correlation, $\mathrm{p}<0.05$ shown in bold).

\begin{tabular}{|c|c|c|c|c|c|c|c|c|}
\hline Items & $\begin{array}{l}3 \\
\mathbb{D} \\
\stackrel{3}{3} \\
\text { क } \\
\text { ב् }\end{array}$ & $\frac{3}{2}$ & 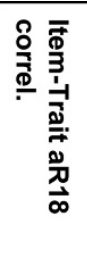 & 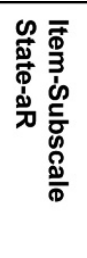 & 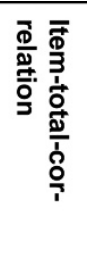 & 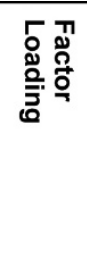 & 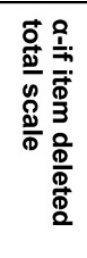 & 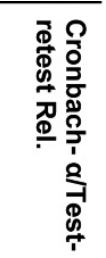 \\
\hline I had cold hands or feet & $\begin{array}{r}3.81 \\
(1.16)\end{array}$ & $1-5$ & 0.24 & 0.45 & 0.35 & 0.556 & 0.816 & \\
\hline I was able to fall asleep & $\begin{array}{r}3.87 \\
(0.96)\end{array}$ & $1-5$ & 0.49 & 0.50 & 0.46 & 0.607 & 0.809 & \\
\hline $\begin{array}{l}\text { I had to pull myself together to do } \\
\text { things } \\
\text { I felt rested in the morning }\end{array}$ & $\begin{array}{r}3.39 \\
(1.13)\end{array}$ & $1-5$ & 0.36 & 0.51 & 0.48 & 0.676 & 0.808 & \\
\hline I suffered from stomach rumbling & $\begin{array}{r}3.89 \\
(1.11)\end{array}$ & $1-5$ & 0.39 & 0.47 & 0.45 & 0.528 & 0.810 & \\
\hline $\begin{array}{l}\text { There were situations where I felt less } \\
\text { competent or skilful than usual }\end{array}$ & $\begin{array}{r}3.80 \\
(1.15)\end{array}$ & $1-5$ & 0.39 & 0.61 & 0.49 & 0.749 & 0.807 & \\
\hline My sleep was restless & $\begin{array}{r}3.58 \\
(1.12)\end{array}$ & $1-5$ & 0.39 & 0.53 & 0.56 & 0.540 & 0.803 & \\
\hline I had cold and sweaty hands or feet & $\begin{array}{r}4.54 \\
(0.79) \\
\end{array}$ & $2-5$ & 0.34 & 0.43 & 0.42 & 0.507 & 0.812 & \\
\hline \multicolumn{9}{|l|}{ S Rest/activity regulation } \\
\hline I felt dizzy when turning round & $\begin{array}{r}4.54 \\
(0.75)\end{array}$ & $2-5$ & 0.46 & 0.61 & 0.52 & 0.770 & 0.809 & $\begin{array}{r}0.805 / \\
0.792\end{array}$ \\
\hline $\begin{array}{l}\text { I felt dizzy when getting up in the } \\
\text { morning }\end{array}$ & $\begin{array}{r}4.58 \\
(0.75)\end{array}$ & $2-5$ & 0.32 & 0.60 & 0.49 & 0.734 & 0.810 & \\
\hline I felt dizzy when turning round quickly & $\begin{array}{r}4.07 \\
(1.07)\end{array}$ & $1-5$ & 0.36 & 0.59 & 0.40 & 0.743 & 0.813 & \\
\hline $\begin{array}{l}\text { I felt dizzy when getting up or bending } \\
\text { down }\end{array}$ & $\begin{array}{r}4.23 \\
(0.82) \\
\end{array}$ & $2-5$ & 0.43 & 0.66 & 0.48 & 0.791 & 0.810 & \\
\hline S Orthostatic-circulatory regulation & $\begin{array}{l}17.41 \\
(2.70)\end{array}$ & $\begin{array}{l}9- \\
20\end{array}$ & 0.48 & 0.60 & 0.68 & & & $\begin{array}{r}0.804 / \\
0.650\end{array}$ \\
\hline I suffered from night sweats & $\begin{array}{r}3.84 \\
(1.25)\end{array}$ & $1-5$ & 0.50 & 0.66 & 0.46 & 0.784 & 0.810 & \\
\hline I sweated & $\begin{array}{r}3.46 \\
(1.07)\end{array}$ & $1-5$ & 0.40 & 0.82 & 0.37 & 0.915 & 0.815 & \\
\hline $\begin{array}{l}\text { I sweated even during light physical } \\
\text { activity }\end{array}$ & $\begin{array}{r}3.75 \\
(1.10) \\
\end{array}$ & $1-5$ & 0.39 & 0.67 & 0.38 & 0.836 & 0.814 & \\
\hline S Thermo-sweating regulation & $\begin{array}{r}11.06 \\
(3.00)\end{array}$ & $\begin{array}{r}3- \\
15\end{array}$ & 0.49 & 0.48 & 0.58 & & & $\begin{array}{r}0.852 / \\
0.601\end{array}$ \\
\hline I had bowel movements & $\begin{array}{r}3.22 \\
(0.95)\end{array}$ & $1-5$ & 0,22 & 0.45 & 0,12 & 0.806 & 0.827 & \\
\hline $\begin{array}{l}\text { I had bowel movements - as always - } \\
\text { at certain times of the day }\end{array}$ & $\begin{array}{r}3.25 \\
(1 ., 37)\end{array}$ & $1-5$ & 0.24 & 0.41 & 0.16 & 0.853 & 0.829 & \\
\hline I suffered from constipation & $\begin{array}{r}4.42 \\
(1.04) \\
\end{array}$ & $1-5$ & 0.32 & 0.65 & 0.38 & 0.615 & 0.814 & \\
\hline S Digestive regulation & $\begin{array}{r}10.89 \\
(2.65) \\
\end{array}$ & $\begin{array}{r}3- \\
14\end{array}$ & 0.29 & 0.69 & 0.44 & & & $\begin{array}{r}0.696 / \\
0.767 \\
\end{array}$ \\
\hline S aR Total Scale & $\begin{array}{l}69.75 \\
(9.25)\end{array}$ & $\begin{array}{r}44- \\
89\end{array}$ & 0.76 & & & & & $\begin{array}{r}0.832 / \\
0.795 \\
\end{array}$ \\
\hline T aR Total Scale & $\begin{array}{l}42.42 \\
(5.26)\end{array}$ & $\begin{array}{r}26- \\
54\end{array}$ & & & & & & $\begin{array}{l}0.741 \\
0.867\end{array}$ \\
\hline T aR Orthostatic-circulatory & $\begin{array}{r}16.49 \\
(6.78) \\
\end{array}$ & $\begin{array}{r}10- \\
21 \\
\end{array}$ & 0.69 & & & & & $\begin{array}{l}0.676 \\
0.803 \\
\end{array}$ \\
\hline T aR Rest/activity & $\begin{array}{r}18.72 \\
(10.9) \\
\end{array}$ & $\begin{array}{r}11- \\
24 \\
\end{array}$ & 0.77 & & & & & $\begin{array}{l}0.697 \\
0.833 \\
\end{array}$ \\
\hline T aR Digestive & $\begin{array}{r}7.26 \\
(1.52)\end{array}$ & $3-9$ & 0.49 & & & & & $\begin{array}{l}0.623 \\
0.879\end{array}$ \\
\hline
\end{tabular}

convergence criteria on the other. Correlation coefficients were determined with Spearman's rank correlations between Trait- and State-aR.

8) We assessed the discriminant validity by applying the exact Mann-Whitney U-test to check whether
State-aR sum- and subscales and Trait-aR are differentiating between cancer and healthy controls, and by comparing both results.

9) We estimated the responsiveness in measuring chemotherapy sensitivity within the $\mathrm{B}$ and $\mathrm{C}$ group 
Table 6. Correlation matrix of the State-aR sum- and subscales and the Trait-aR sum- and subscales with one another and Karnofsky-Index (KPI), A-HADS* \& D-HADS* (*high values more symptoms), Self-regulation scale (SRS), haemoglobin, questions on thermoregulation, EORTC functions and role scales, EORTC Symptoms Scales* (*high values more symptoms) (significant correlation, $\mathrm{p}<0.05$ shown in bold).

\begin{tabular}{|c|c|c|c|c|c|c|c|c|c|}
\hline & 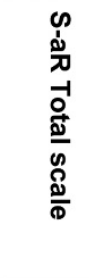 & 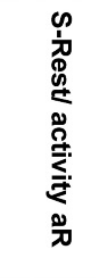 & 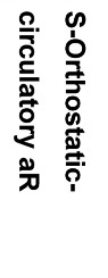 & 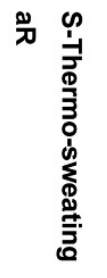 & 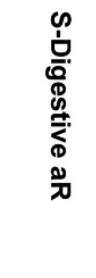 & 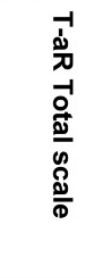 & 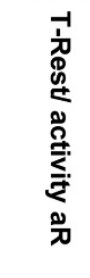 & 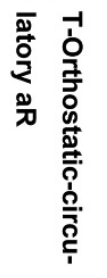 & 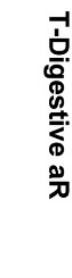 \\
\hline Study 1: S-Rest/activity aR & 0.85 & & & & & & & & \\
\hline $\begin{array}{l}\text { Study 1: S-Orthostatic- } \\
\text { circulatory aR }\end{array}$ & 0.65 & 0.47 & & & & & & & \\
\hline $\begin{array}{l}\text { Study 1: S-Thermo- } \\
\text { sweating aR }\end{array}$ & 0.55 & 0.25 & 0.28 & & & & & & \\
\hline Study 1: S-Digestive aR & 0.36 & 0.12 & 0.02 & 0.09 & & & & & \\
\hline Study 1: T-aR & 0.74 & 0.58 & 0.48 & 0.48 & 0.29 & & & & \\
\hline Study 1: T-Rest/activity & 0.62 & 0.58 & 0.26 & 0.48 & 0.10 & 0.92 & & & \\
\hline $\begin{array}{l}\text { Study 1: T-Orthostatic- } \\
\text { circulatory aR }\end{array}$ & 0.52 & 0.40 & 0.60 & 0.31 & 0.02 & 0.53 & 0.28 & & \\
\hline Study 1: T-Digestive aR & 0.33 & 0.16 & 0.08 & 0.03 & 0.69 & 0.23 & 0.07 & 0.27 & \\
\hline Study 1: KPI & 0.59 & 0.66 & 0.28 & 0.17 & 0.09 & 0.42 & 0.39 & 0.28 & 0.14 \\
\hline Study 1: A-HADS* & -0.44 & -0.41 & -0.33 & -0.31 & -0.31 & -0.55 & -0.53 & -0.43 & -0.15 \\
\hline Study 1: D-HADS* & -0.43 & -0.48 & -0.48 & -0.22 & -0.22 & -0.43 & -0.47 & -0.29 & -0.22 \\
\hline Study 1: SRS & 0.41 & 0.37 & 0.24 & 0.25 & 0.25 & 0.32 & 0.22 & 0.23 & 0.17 \\
\hline Study 1: FNS* & -0.44 & -0.50 & -0.29 & -0.13 & -0.01 & -0.40 & -0.40 & -0.22 & -0.06 \\
\hline Study 1: Haemoglobin & 0.26 & 0.31 & 0.07 & -0.10 & 0.13 & 0.29 & 0.27 & 0.15 & 0.25 \\
\hline $\begin{array}{l}\text { Study 1: Congestive } \\
\text { perspiration }\end{array}$ & 0.41 & 0.29 & 0.26 & 0.43 & 0.05 & 0.31 & 0.39 & 0.19 & -0.03 \\
\hline Study 1: freezing & 0.40 & 0.45 & 0.19 & 0.22 & 0.01 & 0.44 & 0.39 & 0.41 & 0.06 \\
\hline $\begin{array}{l}\text { Study 2: EORTC Physical } \\
\text { funct. }\end{array}$ & 0.40 & 0.49 & 0.14 & 0.25 & 0.02 & 0.34 & 0.36 & 0.20 & -0.21 \\
\hline $\begin{array}{l}\text { Study 2: EORTC Role } \\
\text { funct. }\end{array}$ & 0.27 & 0.23 & 0.10 & 0.21 & 0.17 & 0.22 & 0.21 & 0.08 & -0.07 \\
\hline $\begin{array}{l}\text { Study2: EORTC Emotional } \\
\text { funct. }\end{array}$ & 0.69 & 0.71 & 0.23 & 0.39 & 0.02 & 0.52 & 0.48 & 0.44 & -0.10 \\
\hline $\begin{array}{l}\text { Study 2: EORTC Cognitive } \\
\text { funct. }\end{array}$ & 0.48 & 0.58 & 0.26 & 0.04 & 0.01 & 0.39 & 0.28 & 0.56 & -0.18 \\
\hline $\begin{array}{l}\text { Study 2: EORTC Social } \\
\text { funct. }\end{array}$ & 0.25 & 0.31 & -0.20 & 0.06 & 0.20 & 0.29 & 0.27 & 0.21 & -0.10 \\
\hline $\begin{array}{l}\text { Study 2: EORTC Global } \\
\text { Health }\end{array}$ & 0.52 & 0.50 & 0.14 & 0.19 & 0.30 & 0.43 & 0.39 & 0.21 & 0.09 \\
\hline Study 2: EORTC Fatigue* & -0.35 & -0.35 & -0.20 & -0.08 & -0.04 & -0.24 & -0.18 & -0.21 & 0.07 \\
\hline Study 2: EORTC Dyspnea* & -0.46 & -0.36 & -0.50 & -0.20 & -0.11 & -0.34 & -0.22 & -0.54 & 0.07 \\
\hline Study2: EORTC Sleep & -0.60 & -0.56 & -0.23 & -0.30 & -0.00 & -0.43 & -0.45 & -0.10 & -0.14 \\
\hline
\end{tabular}

with the one-sided Wilcoxon-rank test between first and second and second and third test-point.

10) We tested for superior sensitivity in state-aR over trait-aR by comparing standardized versions of both scales with Wilcoxon signed-rank test.

All analyses were performed with SPSS 16.0 software.

\section{RESUlts STUdy 1 \\ PARTICIPANTS}

We invited 59 patients with malignant conditions and 59 healthy controls to participate. In total, 114 agreed to participate ( $97 \%$ recruitment). From the 57 recruit- 
Table 7. Means and SD for all patients and controls and separated into women and men for the State aR total scale and subscales (State rest/activity, State orthostatic-circulatory, State thermosweating and State digestive regulation) and Trait aR total and subscales (Trait rest/activity regulation, Trait orthostatic-circulatory, Trait digestive regulation). Significant results are shown in bold.

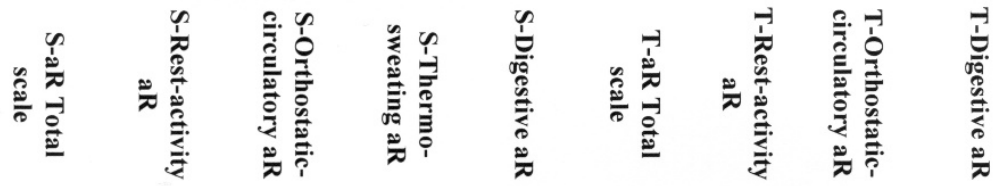

\begin{tabular}{|c|c|c|c|c|c|c|c|c|c|}
\hline \multicolumn{10}{|l|}{ Study 1} \\
\hline \multicolumn{10}{|l|}{ Patients } \\
\hline $\begin{array}{l}\text { Mean all } \\
\text { (SD) }\end{array}$ & $\begin{array}{l}65.86 \\
(9.20)\end{array}$ & $\begin{array}{l}27.81 \\
(5.11)\end{array}$ & $\begin{array}{l}17.12 \\
(2.83)\end{array}$ & $\begin{array}{l}10.61 \\
(3.21)\end{array}$ & $\begin{array}{l}10.30 \\
(2.86)\end{array}$ & $\begin{array}{l}41.49 \\
(5.70)\end{array}$ & $\begin{array}{l}17.85 \\
(3.51)\end{array}$ & $\begin{array}{l}16.57 \\
(2.72)\end{array}$ & $\begin{array}{c}7.00 \\
(1.53)\end{array}$ \\
\hline $\begin{array}{l}\text { Mean women } \\
\text { (SD) }\end{array}$ & $\begin{array}{l}65.24 \\
(8.77)\end{array}$ & $\begin{array}{l}27.51 \\
(4.94)\end{array}$ & $\begin{array}{l}17.00 \\
(2.97)\end{array}$ & $\begin{array}{l}10.07 \\
(2.95)\end{array}$ & $\begin{array}{l}10.63 \\
(2.97)\end{array}$ & $\begin{array}{l}40.54 \\
(5.54)\end{array}$ & $\begin{array}{l}17.59 \\
(3.37)\end{array}$ & $\begin{array}{l}16.00 \\
(2.67)\end{array}$ & $\begin{array}{c}6.95 \\
(1.49)\end{array}$ \\
\hline $\begin{array}{l}\text { Mean men } \\
\text { (SD) }\end{array}$ & $\begin{array}{c}67.44 \\
(10.36)\end{array}$ & $\begin{array}{l}28.56 \\
(5.64)\end{array}$ & $\begin{array}{l}17.44 \\
(2.68)\end{array}$ & $\begin{array}{l}10.56 \\
(3.86)\end{array}$ & $\begin{array}{l}10.87 \\
(2.63)\end{array}$ & $\begin{array}{l}44.14 \\
(5.48)\end{array}$ & $\begin{array}{r}18.53 \\
(3.87) \\
\end{array}$ & $\begin{array}{l}18.07 \\
(2.34)\end{array}$ & $\begin{array}{c}7.12 \\
(1.67)\end{array}$ \\
\hline $\begin{array}{l}\text { Control } \\
\text { Mean all (SD) }\end{array}$ & $\begin{array}{l}73.65 \\
(7.56)\end{array}$ & $\begin{array}{l}32.96 \\
(4.31)\end{array}$ & $\begin{array}{l}17.70 \\
(2.54)\end{array}$ & $\begin{array}{l}11.51 \\
(2.73)\end{array}$ & $\begin{array}{l}11.47 \\
(2.31)\end{array}$ & $\begin{array}{l}43.36 \\
(4.65)\end{array}$ & $\begin{array}{l}19.55 \\
(2.87)\end{array}$ & $\begin{array}{l}16.41 \\
(2.50)\end{array}$ & $\begin{array}{c}7.51 \\
(1.48)\end{array}$ \\
\hline $\begin{array}{l}\text { Mean women } \\
\text { (SD) }\end{array}$ & $\begin{array}{l}73.27 \\
(7.49)\end{array}$ & $\begin{array}{l}32.39 \\
(4.55)\end{array}$ & $\begin{array}{l}17.78 \\
(2.66)\end{array}$ & $\begin{array}{l}11.37 \\
(2.84)\end{array}$ & $\begin{array}{l}11.73 \\
(1.98)\end{array}$ & $\begin{array}{l}42.45 \\
(4.63)\end{array}$ & $\begin{array}{l}18.90 \\
(2.99)\end{array}$ & $\begin{array}{l}16.03 \\
(2.50)\end{array}$ & $\begin{array}{c}7.59 \\
(1.32)\end{array}$ \\
\hline $\begin{array}{l}\text { Mean men } \\
\text { (SD) }\end{array}$ & $\begin{array}{l}74.62 \\
(7.89) \\
\end{array}$ & $\begin{array}{l}34.44 \\
(3.31)\end{array}$ & $\begin{array}{l}17.50 \\
(2.28)\end{array}$ & $\begin{array}{l}10.81 \\
(2.97)\end{array}$ & $\begin{array}{l}11.87 \\
(2.47)\end{array}$ & $\begin{array}{l}42.45 \\
(4.63) \\
\end{array}$ & $\begin{array}{l}18.90 \\
(2.99) \\
\end{array}$ & $\begin{array}{l}16.03 \\
(2.50) \\
\end{array}$ & $\begin{array}{c}7.59 \\
(1.32) \\
\end{array}$ \\
\hline p-value & $<0.001$ & $<0.001$ & 0.21 & 0.14 & 0.015 & 0.090 & 0.006 & 0.75 & 0.029 \\
\hline \multicolumn{10}{|c|}{ Patients vs Control* } \\
\hline \multicolumn{10}{|l|}{ Study 2} \\
\hline $\begin{array}{l}\text { Breast cancer } \\
\text { group } 1 \mathrm{~T} 0\end{array}$ & $\begin{array}{l}70.29 \\
(6.72)\end{array}$ & $\begin{array}{l}30.76 \\
(3.47)\end{array}$ & $\begin{array}{l}17.18 \\
(2.43)\end{array}$ & $\begin{array}{l}10.65 \\
(2.42)\end{array}$ & $\begin{array}{l}11.65 \\
(1.73)\end{array}$ & $\begin{array}{l}1.53 \\
(4.41)\end{array}$ & $\begin{array}{l}17.56 \\
(3.31)\end{array}$ & $\begin{array}{l}16.62 \\
(1.59)\end{array}$ & $\begin{array}{c}7.35 \\
(1.06)\end{array}$ \\
\hline $\begin{array}{l}\text { Breast cancer } \\
\text { group } 1 \mathrm{~T} 1\end{array}$ & $\begin{array}{c}67.50 \\
(10.59)\end{array}$ & $\begin{array}{l}28.94 \\
(6.16)\end{array}$ & $\begin{array}{l}17.19 \\
(3.51)\end{array}$ & $\begin{array}{l}10.81 \\
(2.74)\end{array}$ & $\begin{array}{l}10.50 \\
(2.66)\end{array}$ & $\begin{array}{l}40.25 \\
(6.54)\end{array}$ & $\begin{array}{l}17.50 \\
(3.60)\end{array}$ & $\begin{array}{l}16.06 \\
(3.11)\end{array}$ & $\begin{array}{c}6.76 \\
(1.60)\end{array}$ \\
\hline $\begin{array}{l}\text { Breast cancer } \\
\text { group } 1 \mathrm{~T} 2\end{array}$ & $\begin{array}{l}67.65 \\
(10.09)\end{array}$ & $\begin{array}{l}29.75 \\
(5.93)\end{array}$ & $\begin{array}{l}16.65 \\
(3.82)\end{array}$ & $\begin{array}{l}10.53 \\
(3.20)\end{array}$ & $\begin{array}{l}10.94 \\
(2.68)\end{array}$ & $\begin{array}{l}41.12 \\
(6.53)\end{array}$ & $\begin{array}{l}17.41 \\
(3.69)\end{array}$ & $\begin{array}{l}16.47 \\
(2.87)\end{array}$ & $\begin{array}{l}7.23 \\
(1.39)\end{array}$ \\
\hline $\begin{array}{l}\text { Colorectal cancer } \\
\text { T0 }\end{array}$ & $\begin{array}{l}73.39 \\
(8.62)\end{array}$ & $\begin{array}{l}31.27 \\
(5.24)\end{array}$ & $\begin{array}{l}18.38 \\
(2.28)\end{array}$ & $\begin{array}{l}12.44 \\
(2.48)\end{array}$ & $\begin{array}{l}11.09 \\
(2.88)\end{array}$ & $\begin{array}{l}43.83 \\
(4.13)\end{array}$ & $\begin{array}{l}19.08 \\
(2.86)\end{array}$ & $\begin{array}{l}17.25 \\
(1.77)\end{array}$ & $\begin{array}{c}7.38 \\
(1.64)\end{array}$ \\
\hline Colorectal cancer & 69.87 & 28.46 & 17.46 & 12.42 & 11.58 & 43.29 & 18.81 & 17.04 & 7.57 \\
\hline $\mathrm{T} 1$ & (12.11) & $(6.78)$ & $(3.39)$ & $(3.30)$ & (2.19) & $(5.22)$ & (3.47) & (2.16) & (1.34) \\
\hline $\begin{array}{l}\text { Colorectal cancer } \\
\text { T2 }\end{array}$ & $\begin{array}{l}71.25 \\
(10.04)\end{array}$ & $\begin{array}{l}30.20 \\
(5.99)\end{array}$ & $\begin{array}{l}16.91 \\
(3.45)\end{array}$ & $\begin{array}{l}12.36 \\
(1.91)\end{array}$ & $\begin{array}{l}11.74 \\
(1.42)\end{array}$ & $\begin{array}{l}43.67 \\
(5.13)\end{array}$ & $\begin{array}{l}18.95 \\
(3.42)\end{array}$ & $\begin{array}{l}17.04 \\
(2.07)\end{array}$ & $\begin{array}{c}7.71 \\
(1.00)\end{array}$ \\
\hline $\begin{array}{l}\text { p-value } \mathrm{T} 0 \text { vs } \mathrm{T} 1 \\
* *\end{array}$ & 0.010 & 0.001 & 0.243 & 0.413 & 0.325 & 0.079 & 0.226 & 0.061 & 0.306 \\
\hline $\begin{array}{l}\text { p-value } \mathrm{T} 1 \mathrm{vs} \\
\mathrm{T} 2^{* *}\end{array}$ & 0.484 & 0.061 & 0.106 & 0.395 & 0.114 & 0.243 & 0.432 & 0.222 & 0.119 \\
\hline
\end{tabular}

* Exact U-Test (2-side) between Cancer group and healthy controls,

** Exact Wilcoxon-Test (1-side) used for pooled breast cancer and colorectal cancer and tests T0 (before chemotherapy) vs. T1 (1-5 days after third chemotherapy cycle) and T1 vs. T2 (48 weeks after the end of chemotherapy).

Significant differences $(\mathrm{p}<0.05)$ are shown in bold.

ed cancer patients there were 41 women and 16 men with a mean age of 59.3 years. Age and gender matching with the comparison group was successful (Table 1). Twenty-two patients had no metastases, 35 had a metastatic or generalized disease. The median KPI at the time of recruitment was $90 \%$. The duration of the malignancy was an average of 2.9 years. The mean haemoglobin $(\mathrm{Hb})$ level was $12.1 \mathrm{~g} / \mathrm{dl}(\mathrm{SD}=1.84)$. The questionnaire was re-administered to 65 participants $(57.3 \%)$. Further participant details are listed in Table 2 and a summary of diagnoses in Table 3.

\section{PRETEST ON RELIABILITY}

Because of insufficient item-total correlation and $\alpha$-if item deleted-analysis five items were eliminated from the scale: 'remembering dreams', 'vivid dreams', 'being able to digest big meals', 'feeling well' and 'stool consistency'. This left 18 items in the scale (Table 4).

\section{FIELD COMPLETION}

Apart from the following four items and missing answers, all other fields were completed:

'Feeling dizzy when turning round' (missing answer 1 (a lot)), 'feeling dizzy when getting up in the morning' (a lot), 'feeling dizzy when bending down or getting up' (a lot) and 'cold and sweaty extremities' (a lot). Therefore the answer frequency varies from $0 \%$ to $70.2 \%$ in each case for answers 5 of the questions 'feeling dizzy when turning round quickly' and 'cold and sweaty hands' (Table 4). The mean values vary 
from 3.22 (stool frequency) to 4.58 ('feeling dizzy when turning round quickly').

\section{Principal Component AnAlysis}

Primary factor analysis pointed to a three-principalcomponent model as we achieved for the Trait-aR scale [22]. This model showed an unambiguous, clear factor loading pattern. However, factor one - consisting of the rest/activity and orthostatic-circulatory items - was overwhelmingly dominant compared to the thermo-sweating and digestive factors (12, 3 and 3 items). Yet, although slightly more complex, a fourprincipal components model proved superior with regard to factual and content criteria (comparative factor pattern to the trait scale). Principal component 1 (rest/activity regulation) was analysed by 8 items (range: $8-40$ ) and explained $17.8 \%$ of variance; the second principal component (orthostatic-circulatory regulation) was analysed by 4 items (4-20) which explained $14.5 \%$ of variance; the third component (thermo-sweating regulation) was analysed by 3 items and explained $12.8 \%$ of variance; the fourth component (digestive regulation) was also analysed by 3 items (3-15) and explained $10.8 \%$ of the variance (Tables 4 and 5). In this model, all factors had a minimum eigenvalue close to 2 in the Kaiser criteria and show a largely unambiguous item analysis pattern for the four components. Compared to this the alternatively tested five-principal-components model resulted in a series of ambiguous loading patterns. Therefore the fourprincipal-components model selected was the only convincing model.

\section{ANALYSIS OF RELIABILITY}

The principal results of the reliability analysis were the following:

Item-item-correlations: mean of the correlations is $\mathrm{r}=0.22, \mathrm{~min} / \max =-0.18 / 0.73$, range $=0.91$. The item-variance has a mean of $1.08(\mathrm{~min} / \mathrm{max}=$ $0.56 / 1.86$, range $=1.29)$. The item-total-correlation is $\mathrm{r}_{\mathrm{tr}}=0.12$ (bowel movements frequency) -0.56 (disturbed sleep). The internal consistency measured with Cronbach- $\alpha$ is $r_{\alpha}=0.83$, the test-retest-reliability: $r_{r t}=$ 0.79 ( $p<0.001$ ) (Table 5). The reliability analysis of the subscales show Cronbach- $\alpha$ between $\mathrm{r}_{\alpha}=0.85$ (thermo-sweating regulation) and $\mathrm{r}_{\alpha}=0.70$ (digestive regulation). Factor 3 (thermo-sweating regulation) has the lowest test-retest-reliability with $r_{\mathrm{rt}}=0.60$, the highest was measured for factor 1 (rest/activity regulation, $\left.r_{r t}=0.79\right)$ (Table 5). The subscales correlated with one another $(0.47-0.28)$ with the exception of the digestive regulation subscale $(\mathrm{r}=0.12-0.02)$ (Table 6).

\section{Construct Validity}

All 18 items correlated positively with the Trait-aR scale, ranging from $\mathrm{r}=0.50$ (sweat at night) to $\mathrm{r}=$ $0.22(\mathrm{p}<0.05)$ (bowel movement frequency) (Table 5). The test-retest results of the State-aR with $r=0.60$ (Sthermo-sweating regulation) to $\mathrm{r}=0.79$ (S-rest/activity regulation) are lower compared to those of the
Trait-aR (Trait-aR $\mathrm{r}=0.80$ (T-orthostatic-circulatory $\mathrm{aR})-\mathrm{r}=0.88$ (T-digestive $\mathrm{aR})($ all $\mathrm{p}<0.001)$.

The concurrent validity of the State and the Traitscales ranged from $r=0.48$ to 0.74 (Tables 5,6 ). The following convergence criteria showed positive correlations to high State-aR inclusive of all subscales: low anxiety $(\mathrm{r}=-0.44--0.31)$, low depression $(\mathrm{r}=-0.48-$ $-0.22)$, high self-regulation $(\mathrm{r}=0.41-0.24)$. The following correlated with the sum-scale and the first three subscales: less congestive sweating $(r=0.43-$ $0.26)$ and less 'feeling cold' $(r=0.45-0.19)$ and to the first two subscales and the sum scale: high KPI $(r=$ 0.66-0.28), lower FNS ( $r=0.50-0.29)$. Rest/activity regulation correlated with higher haemoglobin $(\mathrm{r}=$ 0.31 ) and more frequent fevers when suffering from colds $(r=0.20)$.

The sum-scale values distributed from 44 to 89 with a mean value of 69.6; the theoretical minimum/maximum-limits are 18-90. The determination of the discrimination validity was achieved via comparison between the healthy control group and the cancer patients with regards to separation properties between the State-aR-scales compared to the Trait-aR scale (exact U-Test Mann-Whitney: State-aR: $\mathrm{z}=4.4, \mathrm{p}<0.001$; Trait-aR: $z=1.70, p=0.090$, S-rest/activity aR: $z=$ $5.0(p<0.001)$, T-rest/activity aR: $z=2.70(p=0.006)$; S-orthostatic-circulatory aR: $z=1.3(p=0.21)$, T-orthostatic-circulatory: $\mathrm{z}=0.31(\mathrm{p}=0.75)$; S-thermosweating aR: $z=1.5(p=0.14)$; S-digestive $a R: z=$ $2.43(p=0.015)$, T-digestive: $z=2.18(p=0.029)$; therefore the State-aR compared to the Trait-aR shows higher separation properties (Table 7).

\section{Results STUdy 2}

21 patients with breast cancer consented to the study, three declined further participation after their first chemotherapy session, leaving 18 patients who completed the first re-test questionnaire, and one declining the third questionnaire. Therefore we had three completed questionnaires from 17 patients (81\%). 29 patients with colorectal cancer were initially consented to the study, one did not receive chemotherapy, and another declined further participation after their first chemotherapy, session 27 patients completed three questionnaires, before, during and after chemotherapy respectively. $25(86 \%)$ of these could be fully evaluated at all three stages.

State-aR total-scale and rest/activity correlated to varying degrees to HRQL as assessed with the EORTC $(\mathrm{r}=0.70-0.23)$ and symptom scales $(\mathrm{r}=$ $-0.60--0.07)$. Orthostatic-circulatory, thermo-sweating and digestive show just single correlations to the HRQL and symptom scales $(r=-0.56-0.00)$. Of outstanding relevance is the moderate to strong correlation between the sum- and rest/activity scale and the EORTC's "Emotional Functioning" and sleep disturbances $(r=0.71-0.56)($ Table 6$)$.

The pooled breast cancer group and CRC group showed a significant reduction of the State-aR sumand rest/activity scales during chemotherapy while the other State-aR subscales and the Trait-aR scale and subscales show no significant difference (Table 7). We compared the sensitivity of both state and trait-aR 
questionnaires during and outside of chemotherapy treatment. We detected a non-significant increase in sensitivity for the state-aR.

\section{Discussion}

The newly developed scale measuring autonomic regulation as a state (State-aR) relating to the previous week shows good -its subscales a satisfactory to goodinternal consistency [36], which is continuously higher than that of the Trait-Version. The median four week test-retest reliability of the State-aR is $0.05-0.15$ lower than of the Trait-Version, which is coherent with the distinction between state and trait characteristics. The five-week test-retest reliability of the Trait sumscale, rest/activity and orthostatic-circulatory measured in this study is only marginally higher $(0.02-0.15)$ than that of the three-month test-retest reliability of the published Trait-Version [23, 22], which points towards temporal stability of the Trait questionnaire [37].

The State-aR shows a strong correlation with the Trait-aR concurrent test with an $\mathrm{r}=0.74$ and the three parallel subscales $(0.58-0.69)$, consistent with so that a largely comparable construct, although relating to different time frames (previous week/in general). For patients undergoing chemotherapy, the State-aR scale recorded a significant loss of regulation in the sumand rest-activity subscales, compared to the Trait-aR scale. Cross-sectional studies have repeatedly shown a lowered Trait-aR for breast cancer patients vs. healthy individuals $[22,25,23]$. Hence the State-aR captures an increase in sleep problems and problems in daily functioning which is related to fatigue during chemoor radiotherapy [14, 38, 39, 40]. A robust assessment of stability and responsiveness of the Trait-State-aR scale requires further data for validation in patients with other chronic conditions. Compared to other state and trait evaluations such, as for instance, the State and Trait Anxiety Inventory (STAI) the testretest reliability of the State-aR is higher as its questions relate to the preceding week whereas the StateSTAI queries the immediate current state (such as for instance stress experienced at the start of an exam) $[41,37]$. HRQL and self-assessment questionnaires should be clinically relevant and therefore also record a clinically relevant time frame. There is some controversy as to how long this time frame should be [42, 43 ], but the most commonly used time frames range is from one week to four weeks [3, 44].

In order to ensure that the questionnaire construct on State-aR was sufficiently broad we had to accept items with a heterogeneity which capture the different autonomic functions and which had already been validated in the Trait version on aR [22]. This explains one limitation of this validation, that the low item-total correlation for 'bowel movements' $(r=0.12)$ with a low concurrent correlation to the Trait scale of $\mathrm{r}=$ 0.22 [45]. Further limitations are that items such as 'travel sickness', 'dizziness from circular motions' could not be sensibly transformed into a State version relating to the previous week [22]. This is why not all State questions can be matched to identical Trait items. The State-aR scale also differs from the Trait-aR scale with regards to the factor solution as the thermosweating scale is added as the fourth scale [22] which is explained through the additional item 'activity-dependent sweating'.

The four factor solution however still fulfils all common criteria of the test theory with item-subscalecorrelations $r>0.40$, with clear factor loading, good factor differentiation as regards to content as well as satisfactory to good internal consistency [45]. The individual Trait question 'cold and cold and sweaty hands' is split into 'cold hands' and 'cold and sweaty hands' and the sum scale is also supplemented by two items ('fine motor skills', 'activity-dependent sweating'). This, next to a new thermo-sweating subscale, lead to a different weight- and loading pattern: the rest/activity regulation contains more items, including the questions on 'fine motor skills' and the two questions relating to "cold extremities" which, other than in the Trait scale, are no longer included under the section on orthostatic-circulatory regulation [22]. One methodological limitation is that we have calculated test-retest reliability based only on $57 \%$ of all participants in study 1.

Similar to the STAI, where the State-anxiety is described as situational anxiety and Trait-anxiety described as individual, relatively stable anxiety with high test-retest reliability [41], the State-aR can detect a loss of regulation due to illness or also through stressors. The Trait-aR on the other hand can be conceived as a combination of autonomic constitution and current regulation [22]. The new State-aR sum-scale as opposed to the Trait-scale in a cross-sectional context shows a significantly lower aR of the cancer group compared to the control group. In contrast, the Stateas well as the Trait subscales on rest/activity and digestive regulation distinguish between cancer patients and the healthy control group, with the State-scale separating them more clearly. Even, if the sensitivity analysis between both questionnaires yielded to no significant difference between both scales.

The aR-values determined via self-assessment capture autonomic lability (low aR) on the one hand and autonomic stability (high aR) on the other in the sense of an autonomic functioning of the three function scales and the rest/activity scale. High aR correlates low- moderately with the experience of comfort and good thermoregulation (regulated between feeling cold/peripheral vasoconstriction and sweating during heat) $[23,22]$. These results support data describing a strong regulated thermo-regulatory threshold level between perspiration and vasoconstriction, which is dependent not only on circadian rhythm but also on vigilance, personality and gender [46].

The strong correlation of $\mathrm{aR}$ and rest/activity to the KPI is a measure of its convergence validity. The rest/activity regulation and the circadian change of performance orientation during the day (ergotropy) and autonomic order during night sleep (vagotonia) were already recognised to be relevant to health status in the 1960s [47]. We have shown positive correlations of $\mathrm{aR}$ to health and illness for 18 to 93-year-olds in cross-sectional studies $[22,24]$. The low correlation to haemoglobin and moderate correlation to lower anxiety and depression for the State-scale was comparable 
to the Trait subscales [24]. High aR appears to go hand in hand with fewer distress symptoms [48].

The self- regulation scale [27] consists of two subscales entitled 'achieve satisfaction and well-being' and 'change behaviour to reach goal' [28]. The moderate relationship of $\mathrm{aR}$ with self-regulation may indicate both autonomic and psychosomatic interactions. In the case of self-regulation, this is a consistent independent prognostic factor for cancer patients. [27, 49] [50]. Whilst it was not possible to demonstrate an impact of aR on the survival for breast cancer and colorectal cancer patients in contrast to self-regulation [50], aR is a prognostic factor for CRF and cognitive fatigue [51]. The question as to whether aR correlates with the actigraphical measured rest/activity and if it could be a prognostic factor for other chronic conditions awaits the results of ongoing studies.

Our findings suggest that the new questionnaire on State-aR could be more sensitive in detecting the loss of $\mathrm{aR}$ in cancer patients than the Trait-scale. Further research is need to clarify over what period of time the state-aR is more sensitive than the trait-aR scale and to what extent it sensitively measures successful therapy, side effects or progress of malignant conditions. The new scale on State-aR is an instrument which in future can be used to assess adjuvant, palliative and rehabilitative treatments. We are particularly interested in its use in the context of complex oncological, palliative medicine and the effectiveness of complementary medical treatments concepts such as mistletoe or nonpharmacological therapies such as exercise, eurythmy or art-therapy $[52,53]$.

Funding: MK, RZ and MG acknowledge financial support from the Humanus-Institute e.V. Berlin, Germany, MK, FS and MG from Helixor GmbH, Rosenfeld and Abnoba GmbH, Pforzheim, both Germany and FS from Weleda AG, Arlesheim, Switzerland. MK received a grant from the Software AG-foundation, Darmstadt, Germany.

Disclosure and Acknowledgement: Authors declare no conflict of interests.

We thank Anna Schneider, Margarita Kirchhoff, Lisa Arndt, Jenny Puhlemann and Erdmuthe Nickel for recruitment of population, Dagmar Brauer for the database documentation and Thomas Storch for correction of manuscript.

\section{REFERENCES}

1 Ware J, Snow K, Kosinski M et al. SF-36 health survey manual \& interpretation guide. The Health Institute, New England Medical Center, 1993.

2 Aaronson NK, Ahmedzai S, Bergman B et al.: The European Organization for Research and Treatment of Cancer QLQ-C30: A quality-of-life instrument for use in international clinical trials in oncology. J Natl Cancer Inst 1993; 85: 365-376

3 Cella DF, Tulsky DS, Gray G et al.: The functional assessment of cancer therapy scale: Development and validation of the general measure. J Clin Oncol 1993;11:570579.

4 Greisinger AJ, Lorimor RJ, Aday LA et al.: Terminally ill cancer patients. Their most important concerns. Cancer Pract 1997; 5: 147-154.

5 Trask PC, Tellefsen C, Espindle D et al.: Psychometric validation of the cancer therapy satisfaction questionnaire. Value Health 2008; 11: 669-679.
6 Bussing A, Matthiessen PF, Ostermann T: Engagement of patients in religious and spiritual practices: Confirmatory results with the spreuk-p 1.1 questionnaire as a tool of quality of life research. Health Qual Life Outcomes 2005; 3: 53.

7 Stone P, Richardson A, Ream E et al.: Cancer-related fatigue: Inevitable, unimportant and untreatable? Results of a multi-centre patient survey. Cancer fatigue forum. Ann Oncol 2000; 11: 971-975.

8 Hartvig P, Aulin J, Hugerth M et al.: Fatigue in cancer patients treated with cytotoxic drugs. J Oncol Pharm Pract 2006; 12:155-164.

9 Bower JE, Ganz PA, Desmond KA et al: Fatigue in longterm breast carcinoma survivors: A longitudinal investigation. Cancer 2006;106: 751-758.

10 Ahlberg K, Ekman T, Gaston-Johannson F, Mock V: Assessment and management of cancer-related fatigue in adults. Lancet 2003; 362:640-650.

11 Mustian KM, Morrow GR, Carroll JK et al.: Integrative nonpharmacologic behavioral interventions for the management of cancer-related fatigue. Oncologist 2007; 12 Suppl 1:52-67.

12 Davidson JR, MacLean AW, Brundage MD, Schulze K: Sleep disturbance in cancer patients. Soc Sci Med 2002; 54:1309-1321.

13 Arndt V, Merx H, Stegmaier C et al.: Persistence of restrictions in quality of life from the first to the third year after diagnosis in women with breast cancer. J Clin Oncol 2005; 23:4945-4953.

14 Savard J, Villa J, Ivers $H$ et al.: Prevalence, natural course, and risk factors of insomnia comorbid with cancer over a 2-month period. J Clin Oncol 2009; 27:5233-5239.

15 Mormont MC, Waterhouse J, Bleuzen P et al.: Marked 24-h rest/activity rhythms are associated with better quality of life, better response, and longer survival in patients with metastatic colorectal cancer and good performance status. Clin Cancer Res 2000; 6:3038-3045.

16 Muenter NK, Watenpaugh, D. E., Wasmund, W. L. et al.: Effect of sleep restriction on orthostatic cardiovascular control in humans. J Appl Physiol 2000; 88:966972.

17 Farin E, Fleitz A, Follert P: Development of an ICF-orientated patient questionnaire for the assessment of mobility and self-care. Phys Rehab Kur Med 2006; 16:197211.

18 Gutenbrunner C, Fialka-Moser V, Grill E, G S: ICF-coresets in acute settings and early-rehabilitation for patients with musculo-skeletal conditions. Phys Rehab Kur Med 2009;19:14-21.

19 Steiner R: 5.Vortrag vom 25.3.1920; Geisteswissenschaft und medizin biblnr 312. Dornach, Rudolf Steiner Verlag, 1990, vol 6.Auflage, pp. 96-116.

20 Weckenmann M, Kitschmann M, Rauch E et al.: Pilotstudie über die anamnesefragen r.Steiners und ihre beziehung zu klinischen bildern (5.Teil). Merkurstab 1995; 48:417-424.

21 Hoffmann C, Rosenberger A, Troeger W et al.: Validation of questionnaires from several medical fields regarding the constitution of patients. Forsch Komplementarmed Klass Naturheilkd 2002;9:37-44.

22 Kröz M, Feder G, von Laue H et al.: Validation of a questionnaire measuring the regulation of autonomic function. BMC Complement Altern Med 2008; 8:26.

23 Kröz M, Laue von H, Zerm R, Girke M: Development of a questionnaire for endogenous regulation - a contribution for salutogenesis research]. Forsch Komplementär Med Klass Naturheilkd 2003;10:70-77.

24 Kröz M, von Laue H, Zerm R et al.: [Reduction of endogenous regulation in internal medicine patients]. Forsch Komplementarmed Klass Naturheilkd 2005; 12: 333-341. 
25 Kröz M, Linke J, Gutenbrunner C et al.: Cancer fatigue, disturbed rest/activity regulation and sleep disturbances with female patients with non metastatic breast cancer - a pilotstudy. Phys Med Rehab Kuror 2010; 20:152-157.

26 Cohen SR, Mount BM: Living with cancer: "Good" Days and "Bad" Days--what produces them? Can the mogill quality of life questionnaire distinguish between them? Cancer 2000;89:1854-1865.

27 Grossarth-Maticek R: Systemische Epidemiologie und präventive Verhaltensmedizin chronischer Erkrankungen. Berlin, New York, Walter de Gruyter, 1999.

28 Büssing A, Girke M, Heckmann C et al.: Validation of the self regulation questionnaire as a measure of health in quality of life research. Eur J Med Res 2009;14: 223-227.

29 Zigmond AS, Snaith RP: The hospital anxiety and depression scale. Acta Psychiatr Scand 1983;67:361-370.

30 Herrmann C, Buss U: HADS-D, Hospital anxiety and depression scale- Deutsche Version. Testdokumentation und Handanweisung. Bern, Göttingen, Toronto, Seattle, Verlag Hans Huber, 1995.

31 Okuyama T, Tanaka K, Akechi T et al.: Fatigue in ambulatory patients with advanced lung cancer: Prevalence, correlated factors, and screening. J Pain Symptom Manage 2001;22: 554-564.

32 Dittner A, Wessely S, Brown R: The assessment of fatigue. A practical guide for clinicans and researchers. J Psychosom Res 2004;56:157-170.

33 Karnofsky DA, Adelmann W, Craver F: The use of nitrogen mustard in the palliative treatment of carcinoma. Cancer 1948; 1:634-656.

34 Kröz M, Zerm R, Reif M et al.: Validation of a German version of the cancer fatigue scale (CFS-D). European Journal of Cancer Care 2008; 17:33-41.

35 Kröz M, Büssing A, von Laue HB et al: Reliability and validity of a new scale on internal coherence (ICS) of cancer patients. Health and Quality of Life Outcome 2009;7 59

36 Cronbach L: Coefficient alpha and the internal structure of the tests. Psychometrika 1951;16:297-334.

37 Laux L, Glanzmann, P, Schaffner, P, Spielberger, CD: Das State- und Trait-Angstinventar. Theoretische Grundlagen und Handanweisungen. Weinheim, Beltz-Verlag, 1981.

38 Berger AM, Lockhart K, Agrawal S: Variability of patterns of fatigue and quality of life over time based on different breast cancer adjuvant chemotherapy regimens. Oncol Nurs Forum 2009;36:563-570.

39 Fernandes R, Stone P, Andrews P et al.: Comparison between fatigue, sleep disturbance, and circadian rhythm in cancer inpatients and healthy volunteers: Evaluation of diagnostic criteria for cancer-related fatigue. J Pain Symptom Manage 2006; 32:245-254.

40 Ryan JL, Carroll JK, Ryan EP et al.: Mechanisms of cancer-related fatigue. Oncologist 2007;12 Suppl 1:22-34.

41 Spielberger C, Gorsuch R, Lushene R: STAI Manual for the Trait and State anxiety inventory. Palo Alto, CA Consulting Psychologists Press, 1970.

42 King M: Clinical significance in HRQL area; in Bottomley A (ed: Quality of Life, Symptom Research and Patient Reported Outcomes in Cancer Clinical Trials, 2527112009. Brussels, EORTC, 2009,
43 Osoba D, Bezjak A, Brundage M, Pater J: Evaluating health-related quality of life in cancer clinical trials: The national cancer institute of canada clinical trials group experience. Value Health 2007;10 Suppl 2:S138-145.

44 Bullinger M, Kirchberger I: Der sf-36 Fragebogen zum Gesundheitszustand. Göttingen, Hogrefe, 1998.

45 Lienert G, Raatz U: Testaufbau und Testanalyse. Weinheim, Beltz Psychologie Verlags Union, 1998.

46 Tayefeh F, Plattner O, Sessler D et al.: Circadian changes in the sweating-to-vasoconstriction interthreshold range. Eur J Physiol 1998;435:402-406.

47 Hildebrandt G: Leistung und Ordnung. Med Welt 1966;17:2732-2740.

48 Hecht K: Chronobiologische Regulationsdiagnostik zur Verifizierung von funktionellen Zuständen und Dysregulationen; in Hecht K, Scherf H, König O (eds): Emotioneller Stress durch Überforderung und Unterforderung. Berlin, Strasbourg, Schibri, 2001, pp 193-252.

49 Grossarth-Maticek R, Kiene H, Baumgartner SM, Ziegler R: Use of iscador, an extract of european mistletoe (viscum album), in cancer treatment: Prospective non-randomized and randomized matched-pair studies nested within a cohort study. Altern Ther Health Med 2001;7:5766, 68-72, 74-56 passim.

50 Kröz M, von Laue H, Bockelbrink A et al.: Does self regulation and autonomic regulation have an influence on survival in breast and colon carcinoma patients? Results of a prospective study European Journal of Cancer 2009; 7 (supplement 2):192.

51 Kröz M, Zerm R, Kuhnert N et al.: The influence of selfand autonomic regulation on cancer-related fatigue and distress in breast cancer and colorectal cancer patients - a prospective study. European Journal of Integrative Medicine 2009; 1:182.

52 Kienle GS, Kiene H, Albonico HU: [Anthroposophic medicine: Health technology assessment report - short version.]. Forsch Komplementarmed 2006; 13 Suppl 2:718.

53 Büssing A, Ostermann T, Majorek M, Matthiessen PF: Eurythmy therapy in clinical studies: A systematic literature review. BMC Complement Altern Med 2008;8:8.

Received: February 10, 2011 / Accepted: May 23, 2011

Address for correspondence:

Dr. Matthias Kröz

Research Institute Havelhöhe (FIH)

at Community Hospital Havelhöhe

Kladower Damm 221

D-14089 Berlin

Germany

Phone: +49 (0) 30/36501-248

Fax: $\quad+49$ (0) 30/36501-279

E-mail: mkroez@havelhoehe.de 